\title{
Porous 3D graphene aerogel co-doped with nitrogen and sulfur for high-performance supercapacitors
}

Yinan Chen ${ }^{\mathrm{a}}$, Huilian $\mathrm{Hao}^{\mathrm{a}, *}$, Xuekun $\mathrm{Lu}^{\mathrm{b}}$, Wenyao $\mathrm{Li}^{\mathrm{a}, \mathrm{b}, *}$, Guanjie $\mathrm{He}^{\mathrm{c}}$,

Wenzhong Shen ${ }^{\mathrm{d}}$, Paul R. Shearing ${ }^{\mathrm{b}}$ and Dan J. L. Brett ${ }^{\mathrm{b}, *}$

${ }^{a}$ College of Material Engineering, Shanghai University of Engineering Science 333

Long Teng Road, Shanghai 201620, China

${ }^{\mathrm{b}}$ Electrochemical Innovation Lab, Department of Chemical Engineering, University College London, London WC1E 7JE, United Kingdom.

${ }^{\mathrm{c}}$ School of Chemistry, University of Lincoln, Joseph Banks Laboratories, Green Lane, Lincoln, LN6 7DL, United Kingdom.

${ }^{d}$ Institute of Solar Energy, and Key Laboratory of Artificial Structures and Quantum Control (Ministry of Education), Department of Physics and Astronomy, Shanghai Jiao Tong University, 800 Dong Chuan Road, Shanghai 200240, China

*Corresponding author, E-mail: sulee8866@126.com (Huilian Hao); liwenyao314@gmail.com (Wenyao Li); d.brett@ucl.ac.uk (Dan J. L. Brett) 


\begin{abstract}
Heteroatom-doped carbon materials with a high specific area, a well-defined porous structure is important to high-performance supercapacitors (SCs). Here, S and $\mathrm{N}$ co-doped three-dimensional porous graphene aerogel (NS-3DPGHs) have been synthesized in a facile and efficient self-assembly process with thiourea acting as the reducing and doping agent solution. Operating as a SC electrode, fabricated co-doping graphene, i.e., the sample of NS-3DPGH-150 exhibits the highest specific capacitance of $412.9 \mathrm{~F} \mathrm{~g} \mathrm{~g}^{-1}$ under $0.5 \mathrm{~A} \mathrm{~g} \mathrm{~g}^{-1}$ and prominent cycle stabilization with $96.4 \%$ capacitance retention in the back of 10000 cycles. Furthermore, based on NS-3DPGH-150, the symmetrical supercapacitor as-prepared in $6 \mathrm{M} \mathrm{KOH}$ displays a superior energy density of $12.9 \mathrm{Wh} \mathrm{kg}^{-1}$ under the power density of $249 \mathrm{~W} \mathrm{~kg}^{-1}$. Hence, NS-3DPGHs could be considered as an excellent candidate for SCs.
\end{abstract}

Keywords: Porous Structure; Graphene; N, S co-doped; Symmetrical Supercapacitors. 


\section{Introduction}

Supercapacitor (SC) with high power density, fast charging capabilities and long cycling life has desirable characteristics advanced energy storage equipment used in applications like stationary power supply and electric vehicles [1,2]. The electrode material is arguably the most crucial SC component and intensive research continues to discover and optimize novel electrode materials that improve the high-rate capability, specific capacitance, energy density and longevity. It is widely acknowledged that maximizing surface area and optimizing the hierarchical structure of electrode materials, i.e., the three-dimensional (3D) porous structure, is one of the most effective approaches to obtain high supercapacitor property [3, 4].

Graphene, with high electronic conductivity, good electrochemical stability, excellent specific surface area and has been confirmed extensively for a promising supercapacitor electrode material. However, two-dimensional (2D) graphene easily undergoes restacking and agglomeration through strong hydrophobic interaction and van der Waals interactions between graphene sheets diminish high surface area and suppress practical applications of 2D graphene [5-7]. Self-assembling 2D graphene into 3D interconnected porous structures, especially hydrogels and aerogels, is an effective way to obtain a porous structure, leading to outstanding electrochemical performance [8-10]. Incorporating heteroatoms such as $\mathrm{N}$ or $\mathrm{S}$ into graphene can improve surface properties, which is desirable for electrochemical energy storage [11-14]. For instance, introducing nitrogen atoms into graphene not only introduces nitrogen-containing functional groups into graphene to improve hydrophilicity that is helpful for the electrolyte infiltration and contribute pseudocapacitance, but also improves electronic conductivity $[15,16]$. Furthermore, nitrogen-doped graphene has a modified electronic structure that can be transferred to electrochemical sites with a 
minimized variation in conjugated length because of charge polarization [17]. By doping $\mathrm{S}$ atoms into graphene, active sites and structural defects can be generated due to the disruption of electron density balance and the relatively large size of sulfur, respectively, which make these materials have unique electronic structures and high specific surface areas [18]. In sulfur-doped carbon materials, negatively charged sulfur atoms have P-orbitals in the outermost layer and have an electron structure similar to that of nitrogen atoms [19]. So, it is supposed that the simultaneous introduction of both $\mathrm{S}$ and $\mathrm{N}$ into graphene could be an effective approach to enhance electrochemical capacitance. The N/S co-doped graphene imparts defected morphology and a favorable electronic structure. After the co-doped, Faradaic redox reactions occur on pyrrole and pyridine groups and increasing sulfones and sulfoxides species to increase the pseudo capacitance [20].

In this work, a scalable approach that uses thiourea as the combined reducing and doping agent in graphene oxide solution to fabricated $\mathrm{S}$ and $\mathrm{N}$ dual-doping three-dimensional porous graphene aerogel (GA) (NS-3DPGHs) is reported. The unique 3D porous structures of NS-3DPGHs provide greatly specific areas and shorter transport routes for the diffusion of ion, leading to wonderful electrochemical performance. Depending on the amount of thiourea used in the synthesis, the binder-free NS-3DPGH electrodes obtained exhibit different electrochemical performance. NS-3DPGH-150 was found to exhibit the highest supercapacitor capacitance (412.9 $\mathrm{F} \mathrm{g} \mathrm{g}^{-1}$ under $0.5 \mathrm{~A} \mathrm{~g}^{-1}$ ) and preferable cyclical stabilization $(96.4 \%$ capacitance retention at the back of 10000 periods), as well as the superior energy density of $12.9 \mathrm{Wh} \mathrm{kg}^{-1}$ under the power density of $249 \mathrm{~W} \mathrm{~kg}^{-1}$ when fabricated into the symmetric SC.

\section{Experimental}




\subsection{Fabrication of materials}

Graphene oxide (GO) was fabricated using advanced Hummers' approach [21]. Pour $960 \mathrm{mg}$ GO powder into $240 \mathrm{~mL}$ deionized water and ultrasonic stirring for 30 minutes. Thiourea $(50,100,150$ and $200 \mathrm{mg}$ ) was added into $60 \mathrm{~mL}$ of $\mathrm{GO}$ dispersion to prepare $\mathrm{S}$ and $\mathrm{N}$ dual-doped graphene, which was then transferred into a $100 \mathrm{~mL}$ Teflon-lined stainless steel autoclave. Finally, the system was heated and sealed at $180{ }^{\circ} \mathrm{C}$ for $24 \mathrm{~h}$. After being purified by deionized water and ethanol, the hydrogel was freeze-dried $48 \mathrm{~h}$ until naturally cooling to room temperature. Resultant samples termed 'NS-3DPGH-X', where $\mathrm{X}$ represents the thiourea mass. In addition, undoped GA was prepared without added thiourea under the same experimental conditions.

\subsection{Characterization}

The X-ray diffraction (XRD) patterns of the samples were examined on a Panalytical X' Pert X-ray diffractometer (Holland). Raman spectra were obtained by a Via Raman microscope (Renishaw, England). The microstructure and porous morphology of the samples were examined using scanning electron microscopy (SEM, FEI, Holand) and high-resolution transmission electron microscopy (HRTEM, JEOL JEM-2100F, Japan). The $\mathrm{N}_{2}$ adsorption-desorption isotherm measurements of the samples were performed using a Micromeritics ASAP 2020 (America) analyzer. The specific surface area was determined using the Brunauer-Emmett-Teller (BET) method, where the samples were degassed under vacuum at $160{ }^{\circ} \mathrm{C}$ for $4 \mathrm{~h}$ before the measurement. The chemical compositions of the carbon materials were quantitatively analyzed by X-ray photoelectron spectroscopy (XPS, Physical Electronics 5400 ESCA).

\subsection{Electrochemical measurements}

The working electrode was fabricated by pressing $4 \mathrm{mg}$ of NS-3DPGH onto a 
precleaned nickel foam substrate $\left(1.0 \times 1.0 \mathrm{~cm}^{2}\right)$ under $10 \mathrm{MPa}$ for $30 \mathrm{~s}$. The electrochemical measurements were performed in a three-electrode system by using an electrochemical workstation ( $\mathrm{CHI} 660$, Shanghai), containing $\mathrm{Ag} / \mathrm{AgCl}$ as the reference electrode and a platinum foil as the counter electrode. The cyclic voltammetry (CV) was tested at scan rates of $5 \mathrm{mV} \mathrm{s}^{-1}$ to $200 \mathrm{mV} \mathrm{s}^{-1}$ in the voltage range from -1 to $0 \mathrm{~V}$. Galvanostatic charge-discharge (GCD) and cycle performance were also conducted from -1 to $0 \mathrm{~V}$. The electrochemical impedance spectroscopy (EIS) was performed with $5 \mathrm{mV}$ amplitude in a frequency range from $100 \mathrm{kHz}$ to 0.01 Hz. $6 \mathrm{M} \mathrm{KOH}$ solution was used as the electrolyte in all electrochemical tests. Two pieces of work electrodes of the same load mass were immersed in $6 \mathrm{M} \mathrm{KOH}$ solution for 6 hours, which acted as positive and negative electrodes of SC, respectively. The cellulosic paper was performed as a diaphragm between the two work electrodes, which were fixed by the conductive adhesive between two polypropylene separators. The separators were fixed by acid and alkali resistant screws.

\section{Results and discussion}

\subsection{Microstructure characterizations}

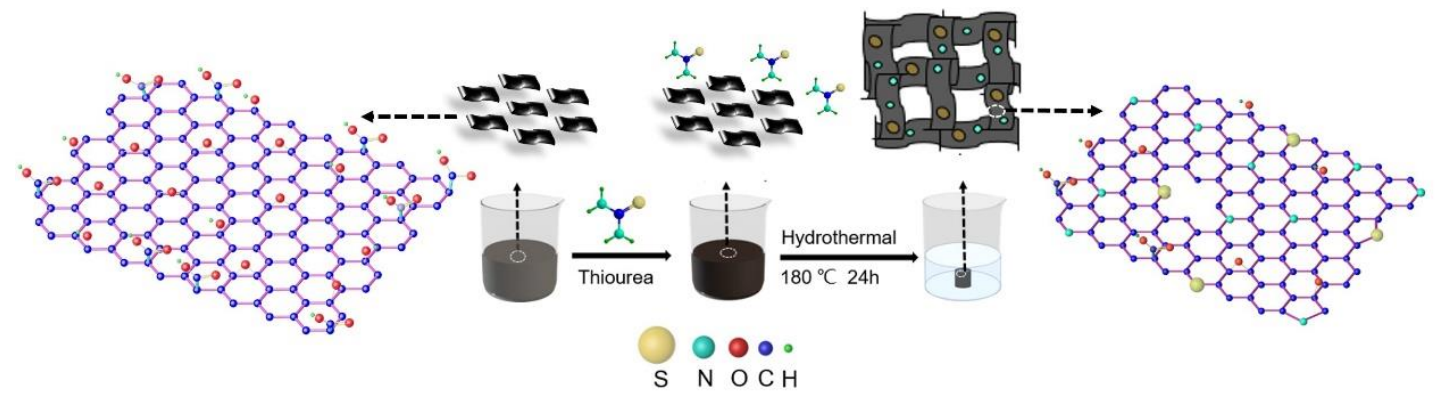

Figure 1. Graphical scheme of the synthetic process of NS-3DPGH.

A scheme illustrating the synthetic procedure of NS-3DPGH is shown in Figure 1. Thiourea served as the nitrogen $(\mathrm{N})$ and sulfur $(\mathrm{S})$ source and also acted as the 
reducing agent. During the mixing process, thiourea becomes associated with the oxygen-containing groups on $\mathrm{GO}$ by hydrogen $(\mathrm{H})$ bonds or electrostatic interactions. Then, during the hydrothermal process $\left(180{ }^{\circ} \mathrm{C}, 24\right.$ hours), the $\pi-\pi$ reciprocities existing self-assembly process resulted in 3D porous formation.
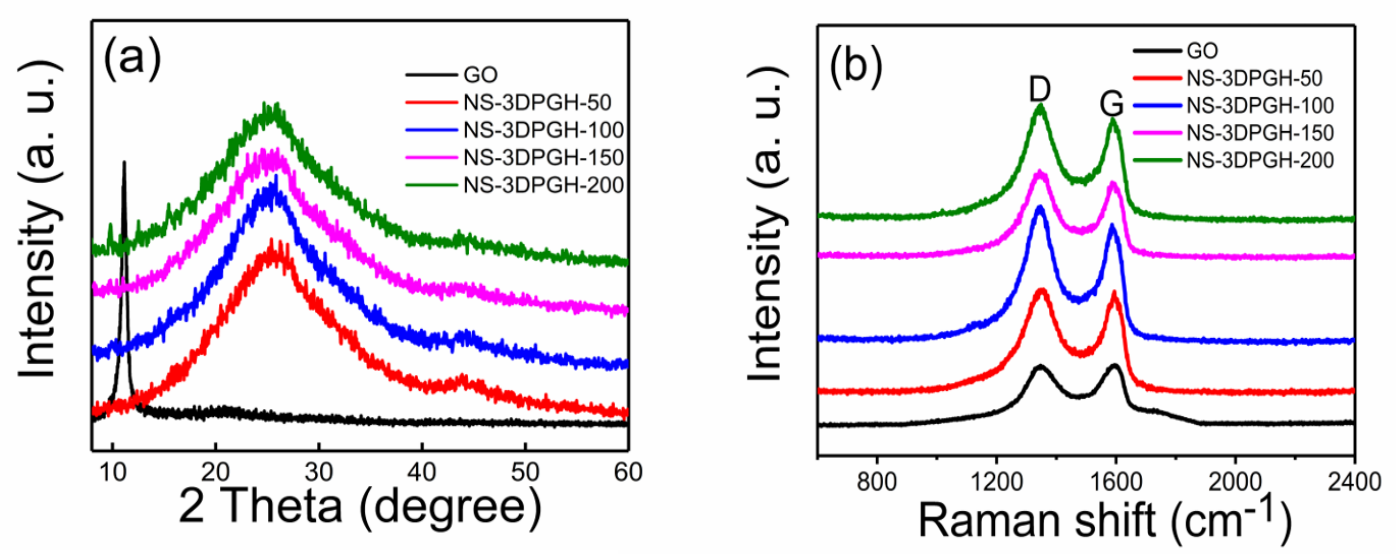

Figure 2. (a) XRD patterns of NS-3DPGHs and GO (b) Raman spectra of NS-3DPGHs and GO.

XRD was performed to verify the transformation from GO to NS-3DPGHs (Figure 2a). The peak at $10.5^{\circ}$ disappeared, meanwhile, the new broadened peaks emerge at $25.4^{\circ}$ which refers to (002) plane of graphite after the hydrothermal process for NS-3DPGH, suggesting the successful reduction of GO. Based on the Bragg equation, the calculated d-spacings for NS-3DPGH-50, NS-3DPGH-100, NS-3DPGH-150 and NS-3DPGH-200 are 3.60, 3.71, 3.55 and $3.76 \AA$, respectively. All of the values are lower than that of GO, indicating the recovery of the natural graphite. Also, the peaks of NS-3DPGHs are broader and weaker than GO, which can be ascribed to the abundant pores in the modified graphene [23].

Figure $2 b$ exhibits the typical Raman spectra of NS-3DPGH and GO. It is observed that two prominent peaks at about $1580 \mathrm{~cm}^{-1}$ and $1350 \mathrm{~cm}^{-1}$ which ascribed to G-band 
(the $\mathrm{E}_{2 \mathrm{~g}}$ mode of $\mathrm{sp}^{2}$ carbon atoms) and D-band (the $\mathrm{A}_{1 \mathrm{~g}}$ symmetry mode) are shown for all samples. Generally, the value of intensity ratio $\left(I_{D} / I_{G}\right)$ for $\mathrm{D}$ to $\mathrm{G}$ band is applied to estimate defects of carbon materials. As can be seen from Table S1, the value of $I_{D} / I_{G}$ for NS-3DPGH-150 was higher than other samples because of the lowest d-spacing value of XRD analysis. The values of $I_{D} / I_{G}$ for NS-3DPGHs are higher than GO, which suggests increased defects caused by heteroatom doping and diminish in size for the re-built graphene meshwork ( $\mathrm{sp}^{2}$ domain), which is same with the previous reports [22-26].

Figure 3 displays the SEM images of NS-3DPGHs and TEM images of NS-3DPGH-150, all of which show typical continuous 3D network structures composed of graphene sheets physical cross-linking. The distinct structures of NS-3DPGHs can be ascribed to the partial overlapping of the wrinkled graphene, through $\pi-\pi$ stacking interaction. Meanwhile, the difference between the morphologies and internal structure of the NS-3DPGHs with different thiourea was further observed. With the mass of the thiourea increasing from 50 to $200 \mathrm{mg}$, the size of the interconnected pores gradually increases from a few micrometers to tens of micrometers, meanwhile some smaller pores within the 'silk-like' textures form the characteristic hierarchical structure. The unique three-dimensional porous structure is beneficial to fast electrolyte permeation and ion transport [27-30]. Representative TEM images of NS-3DPGH-150 are displayed in Figure 3e-f to provide further information on the morphology of structures. The distinct interconnected porous structure and the small thickness of graphene can be seen both at high and low magnifications. Such a unique structure is considered to be beneficial for supercapacitor-based electrochemical energy storage. Elemental mapping images are shown in Figure $3(\mathrm{~g}-\mathrm{k})$ to visualize the element distribution. As expected, the $\mathrm{O}, \mathrm{S}, \mathrm{N}$, 
and $\mathrm{C}$ elements are homogeneously dispersed in the matrix, indicating $\mathrm{S}$ and $\mathrm{N}$ are successfully doped.

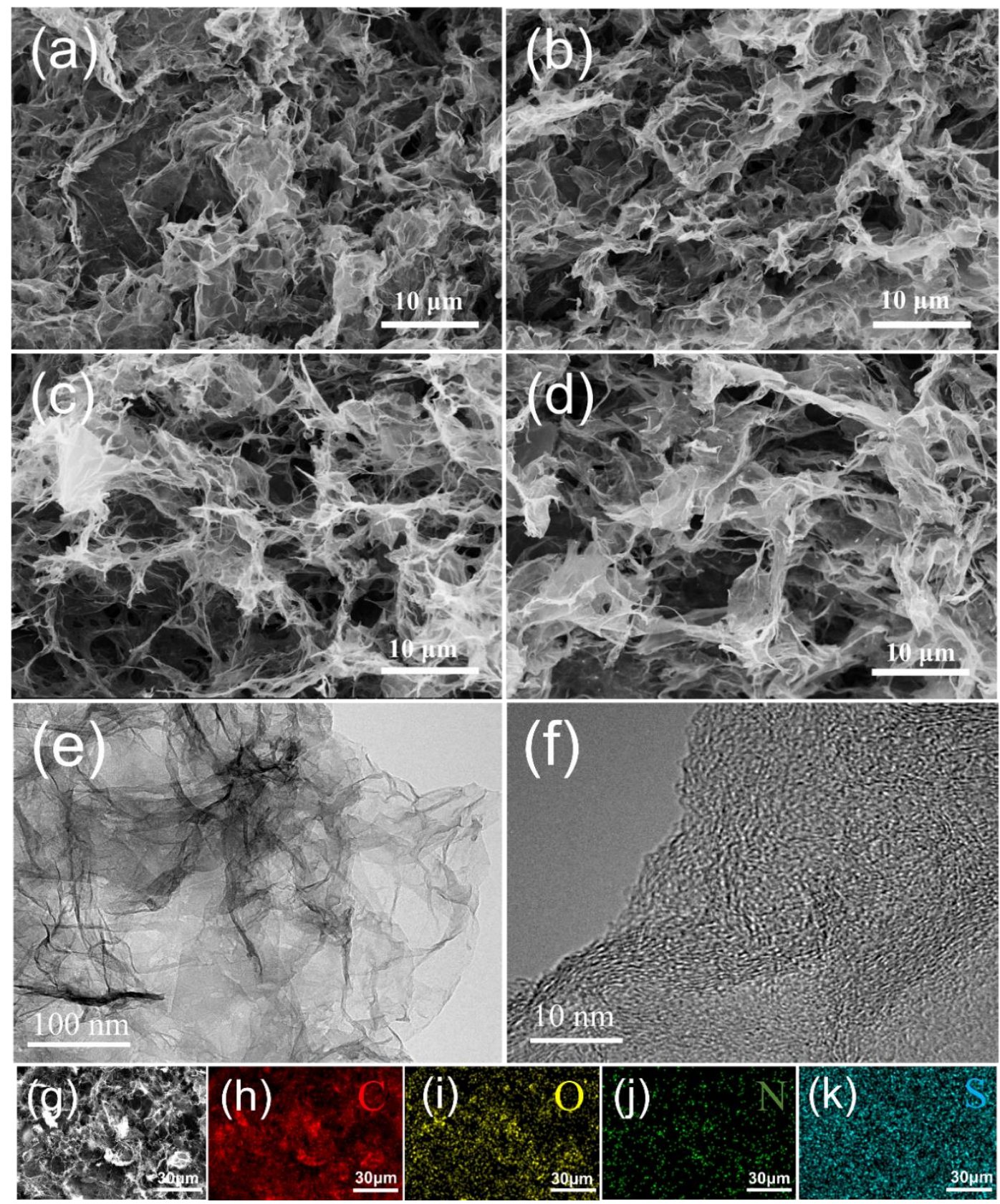

Figure 3. SEM images of the NS-3DPGH-50 (a), NS-3DPGH-100 (b), NS-3DPGH-150 (c) and NS-3DPGH-200 (d). The TEM images of NS-3DHPGH-150 at different magnifications (e-f). EDS mapping of electron image of the selected area of NS-3DPGH-150 (g). Elemental-mapping images of $\mathrm{N}, \mathrm{O}, \mathrm{C}$ and $\mathrm{S}$ of NS-3DPGH-150 (h-k). 

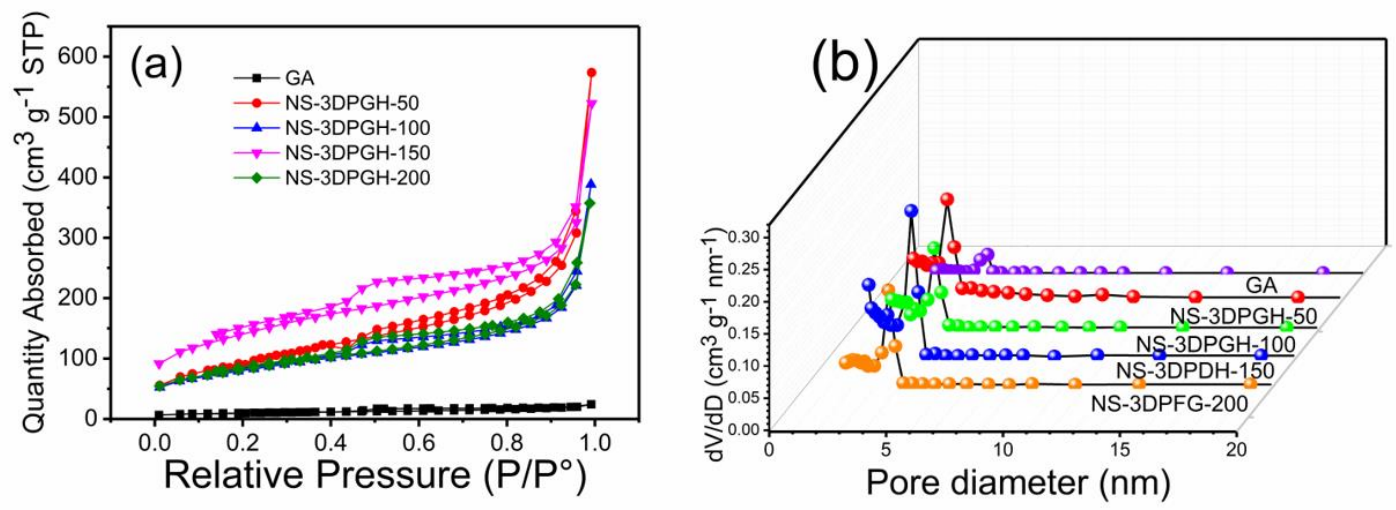

Figure 4. (a) $\mathrm{N}_{2}$ adsorption/desorption isotherms for NS-3DPGHs and GA. (b) The corresponding micropore size distribution calculated from the adsorption isotherms using the Barrett-Joyner-Halenda (BJH) method.

The porosity is a critical element to determine the performance of SC electrodes. $\mathrm{N}_{2}$ adsorption/desorption isotherm measurements were taken to characterize the porous structure of NS-3DPGHs. In Figure 4a, Type-IV isotherm with hysteresis loops can be observed from the curves of NS-3DPGHs. After the doping, the absorption volume of the NS-3DPGHs has significantly increased compared with that of the GA, suggesting that specific surface area (SSA) could be improved by N and S co-doped. The SSA of NS-3DPGH-150 is tested to be $498.18 \mathrm{~m}^{2} \mathrm{~g}^{-1}$, which is better than the other NS-3DPGHs and GA (Supporting Information, Table S2). It is more beneficial to ionic exchange to facilitate charge migration with higher SSA. The pore size distribution of NS-3DPGHs and GA derived from the BJH method (Figure 4b) is observed to primarily fall between 2-4 nm, implying the existence of mesopores and is the same as consequences in Figure 3. Besides, the total pore volume of GA is smaller than NS-3DPGHs, suggesting a high SSA and fast ion transportation. Accordingly, the high SSA and porosity of NS-3DPGH-150 with abundant mesopores is advantageous in facilitating the electrolyte ions diffusion, thus enhancing electrochemical capacitance [28-29]. 

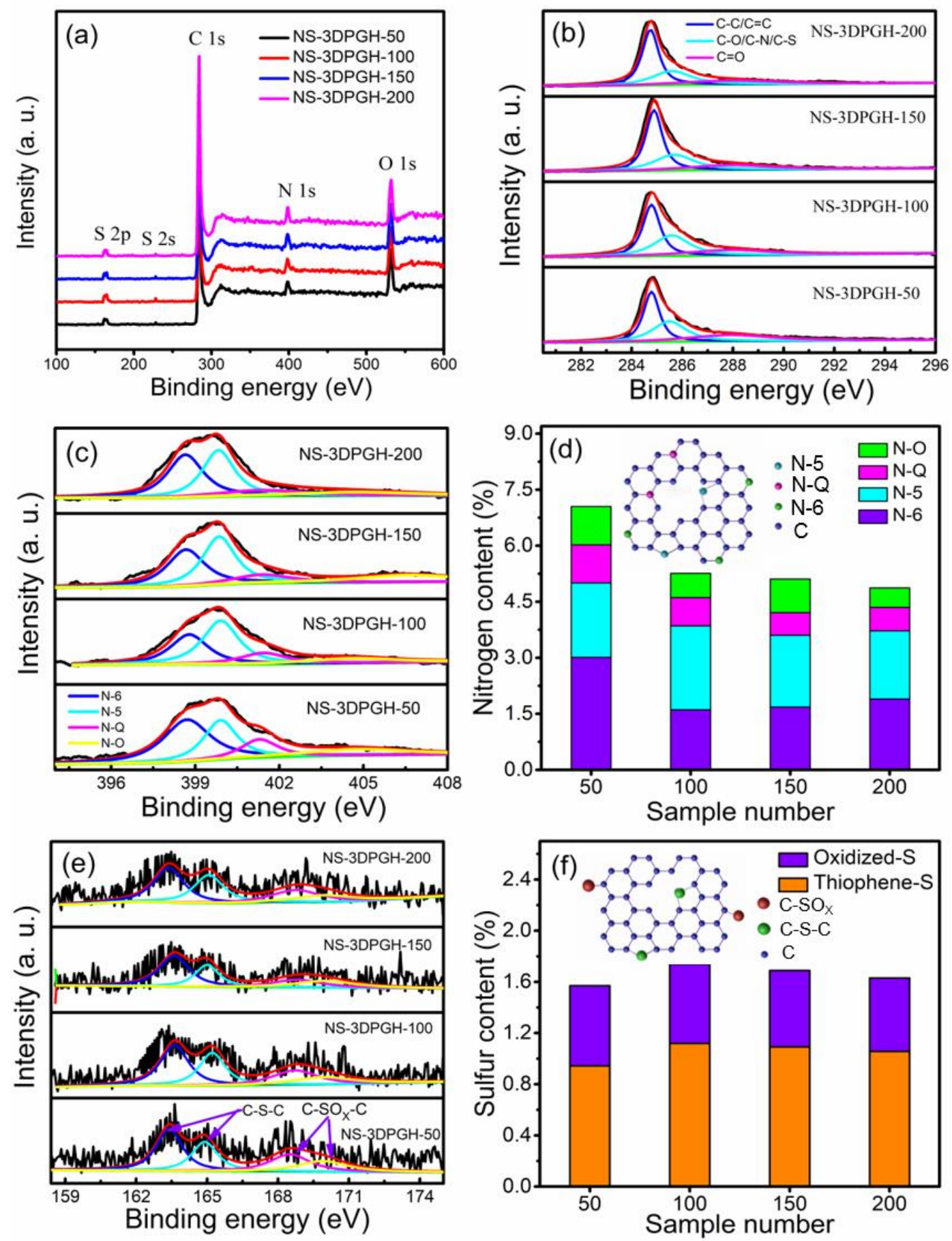

Figure 5. XPS spectra of (a) survey scan. (b) C 1s. (c) N 1s for NS-3DPGHs. (d) Contents of four kinds of $\mathrm{N}$ in NS-3DPGHs. (e) $\mathrm{S} 2 \mathrm{p}$ spectra of NS-3DPGHs. (f) Contents of two kinds of S in NS-3DPGHs.

XPS measurements were conducted to advanced survey examine surface chemical composition. The survey spectrum of the NS-3DPGHs presents peaks at 163.9, 229.0, 
284.5, 399.8 and $532.2 \mathrm{eV}$ (Figure 5a), corresponding to S 2s, S 2p, C 1s, N 1s, and O 1s, respectively. The existence of $\mathrm{S}$ and $\mathrm{N}$ elements in NS-3DPGHs confirms that GA has been successfully doped. The high-resolution C 1s spectra of NS-3DPGHs in Figure $5 \mathrm{~b}$ displays the peaks of, $\mathrm{C}-\mathrm{C}$ and $\mathrm{C}=\mathrm{C}$ in $\mathrm{sp}^{2}$-hybridized domains $(284.8 \mathrm{eV})$, C-O/C-N/C-S $(285.5 \mathrm{eV})$, and $\mathrm{C}=\mathrm{O}(287.8 \mathrm{eV})$, respectively [31]. The peak of $\mathrm{C}=\mathrm{O}$ suggests that the presence of residual carboxyl and carbonyl groups, which decrease with the increase of the thiourea mass, suggesting that the deoxygenation of GO is dependent on the content of thiourea. Nitrogen content is within the limits of 4.87-7.05 at.\%, which gradually increases with the thiourea content increasing (Table S1). As clearly shown in Figure 5c, the $\mathrm{N}$ 1s spectra of NS-3DPGHs exhibit the partial conversion of nitrogen atoms to the pyridinic $\mathrm{N}(\mathrm{N}-6$ at $398.7 \mathrm{eV})$, pyrrolic $\mathrm{N}$ $(\mathrm{N}-5$ at $399.9 \mathrm{eV})$, graphitic $\mathrm{N}(\mathrm{N}-\mathrm{Q}$ at $401.3 \mathrm{eV})$ and oxidized $\mathrm{N}(\mathrm{N}-\mathrm{O}$ at $404.8 \mathrm{eV})$ forms [32]. This confirms that $\mathrm{N}$ has been doped into the graphene framework successfully. The relative amount of each $\mathrm{N}$ species is graphically summarized in Figure 5d. It can be observed that the types of $\mathrm{N}$ groups have principally existed as the N-5 and N-6. The introduction of N-6 and N-5 can endow the carbonaceous materials with pseudocapacitance via proton incorporation. Moreover, N-Q is come from a $\mathrm{N}$ atom replacing for a $\mathrm{C}$ atom in a hexagon and it is of great significance to improve the hydrophilicity and enhance the electrical conductivity [33-35].

Figure 5e exhibits the well-fitted S 2p spectra of NS-3DPGHs and the content of S in the range of $1.57-1.74$ at.\% is shown in Table $\mathrm{S} 1$. The large sulfur radius makes it more difficult to be embedded in the framework, which explains the reason for the relatively low sulfur content compared to nitrogen content in the samples. The $S 2 p$ was divided into four constituents. It is obtained that two peaks located at 163.4 and $164.9 \mathrm{eV}$ are assigned to $\mathrm{S} 2 \mathrm{p}_{3 / 2}$ and $\mathrm{S} 2 \mathrm{p}_{1 / 2}$ of thiophene sulfur (C-S-C), respectively. 
Moreover, the other two peaks centered at 169.9 and 168.5 are ascribed to oxygen-containing sulfur. It can be found that the varieties of thiophene sulfur become foremost with increasing the content of thiourea, as exhibited in Figure $5 \mathrm{f}$. It is reported that the doped sulfur can improve the carbon materials' surface properties, thus providing more defects and promoting the electrolyte permeation that enhances the electrochemical properties [31]. The XPS results confirm that sulfur and nitrogen atoms doped into the graphene framework successfully.

To further confirm that $\mathrm{N}$ and $\mathrm{S}$ atoms have been doped in the carbon skeletons. The FTIR spectra of GO and NS-3DPGH-150 are shown in Figure S1. The peak at $1643 \mathrm{~cm}^{-1}, 1739 \mathrm{~cm}^{-1}$ and $3470 \mathrm{~cm}^{-1}$ are related to the $\mathrm{C}=\mathrm{C}, \mathrm{C}=\mathrm{O}$ stretching vibration and hydroxyl group $(\mathrm{O}-\mathrm{H})$, respectively. The peak at $830 \mathrm{~cm}^{-1}$ is ascribed to the $-\mathrm{NH}_{2}$ and $\mathrm{C}-\mathrm{N}$ bond. The peak at $1378 \mathrm{~cm}^{-1}$ representing $\mathrm{C}-\mathrm{N}$ stretching vibration appears in the spectra of NS-3DPGH-150, indicating the successful incorporation of $\mathrm{N}$ atoms in the hydrothermal process. The peak at $640 \mathrm{~cm}^{-1}$ represents C-S stretching vibration, implying the $\mathrm{N}$ and $\mathrm{S}$ atoms have been successfully doped in the carbon skeletons $[10]$.

3.2. Electrochemical performance of NS-3DPGH 

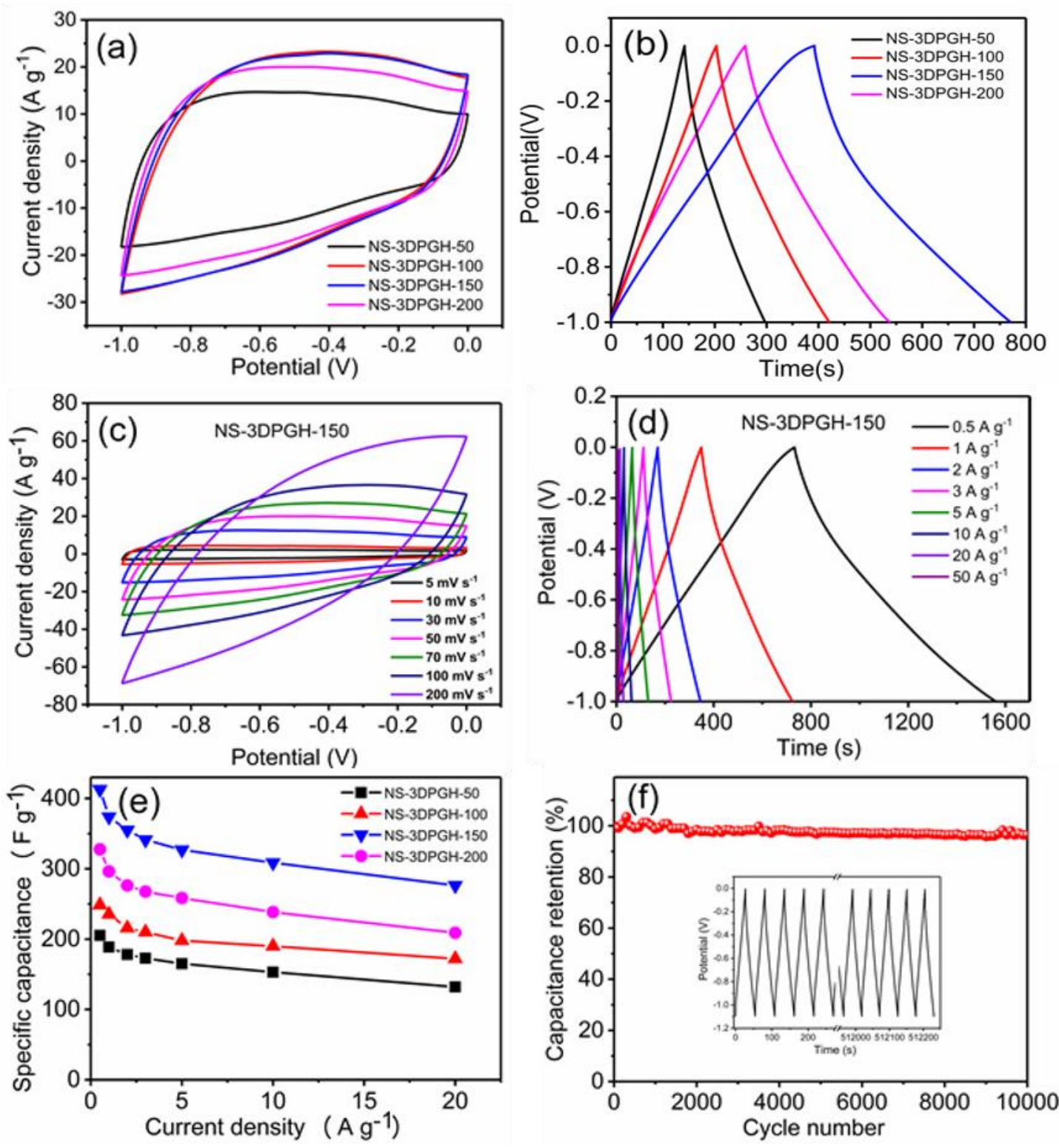

Figure 6. (a) CV-curve of NS-3DPGHs under a scan rate of $50 \mathrm{mV} \mathrm{s}^{-1}$. (b) GCD-curve of NS-3DPGHs under $1 \mathrm{~A} \mathrm{~g}^{-1}$. (c) CV-curve of NS-3DPGH-150 under various scan rates. (d) GCD-curve of NS-3DPGH-150 under various current densities. (e) Specific capacitance under different current densities. (f) Long-term cycling test of NS-3DPGH-150 at $10 \mathrm{~A} \mathrm{~g}^{-1}$.

The electrochemical performance of NS-3DPGHs as an active electrode material was explored by $\mathrm{CV}$ and GCD in three-electrode systems with a $6 \mathrm{M} \mathrm{KOH}$ aqueous solution electrolyte. The corresponding CV curves of the NS-3DPGHs obtained at the 
scan rate of $50 \mathrm{mV} \mathrm{s}^{-1}$ are displayed in Figure $6 \mathrm{a}$. All CV curves of four samples show approximate rectangular-like shape, illustrating that the double-layer (DL) capacitance is the primary charge storage mechanism. The slightly asymmetric form of the curves is ascribed to pseudocapacitance related to the heteroatoms [36]. The NS-3DPGH-150 displays the largest $\mathrm{CV}$ curve area, suggesting that this formulation has the highest specific capacitance. The GCD curves of NS-3DPGHs at the same current density of $1 \mathrm{~A} \mathrm{~g} \mathrm{~g}^{-1}$ are exhibited in Figure 6b. A high level of nearly linear and symmetry discharge slopes, without appreciable voltage drop, are observed for all curves; this is indicative of DL capacitance with a small internal resistance of the NS-3DPGHs electrodes. According to the CV result, the charge-discharge time of NS-3DPGH-150 is the longest, indicating that NS-3DPGH-150 has the largest specific capacitance.

The CV curves of NS-3DPGH-150 at different scan rates are displayed in Figure 6c. Even when the scan rate increases to $200 \mathrm{mV} \mathrm{s}^{-1}$, the shape of the $\mathrm{CV}$ curve still retains rectangular-like, indicating excellent rate performance. The different scan rates of GA of CV curves are displayed in Figure S2a. Figure 6d presents the GCD curves of NS-3DPGH-150 at different current densities are presented. These curves exhibit a triangle shape with slight arcs in the charge-discharge process, indicating predominantly DL capacitance behavior. Figure 6d and S2b show the capacitance of NS-3DPGH-150 is much higher than that of GA. The slight asymmetry is attributed to additional pseudocapacitance owing to the incorporation of $\mathrm{N}$ and $\mathrm{S}$.

Based on Eq. (2) in Supporting Information, the specific capacitance is exhibited in Figure 6e. NS-3DPGH-150 reaches a high specific capacitance of $412.9 \mathrm{~F} \mathrm{~g}^{-1}$ under a current density of $0.5 \mathrm{~A} \mathrm{~g}^{-1}$, which is significantly higher than many $\mathrm{N}$ and $\mathrm{S}$ co-doped carbon electrodes reported previously, as displayed in Table S3. Even at a high current density of $20 \mathrm{~A} \mathrm{~g}^{-1}$, NS-3DPGH-150 possesses a specific capacitance of $262.6 \mathrm{~F} \mathrm{~g}^{-1}$, 
implying excellent rate performance (63.6\% retention). It is noticeable all the electrodes experience obvious capacitance loss with increasing current density, which could be caused by ion transport limitation through the porous network or decrease in the pseudocapacitive reaction. However, NS-3DPGH-150 shows $96.4 \%$ capacitance retention after 10000 cycles (Figure 6f), which indicates good long-term cycling stability.
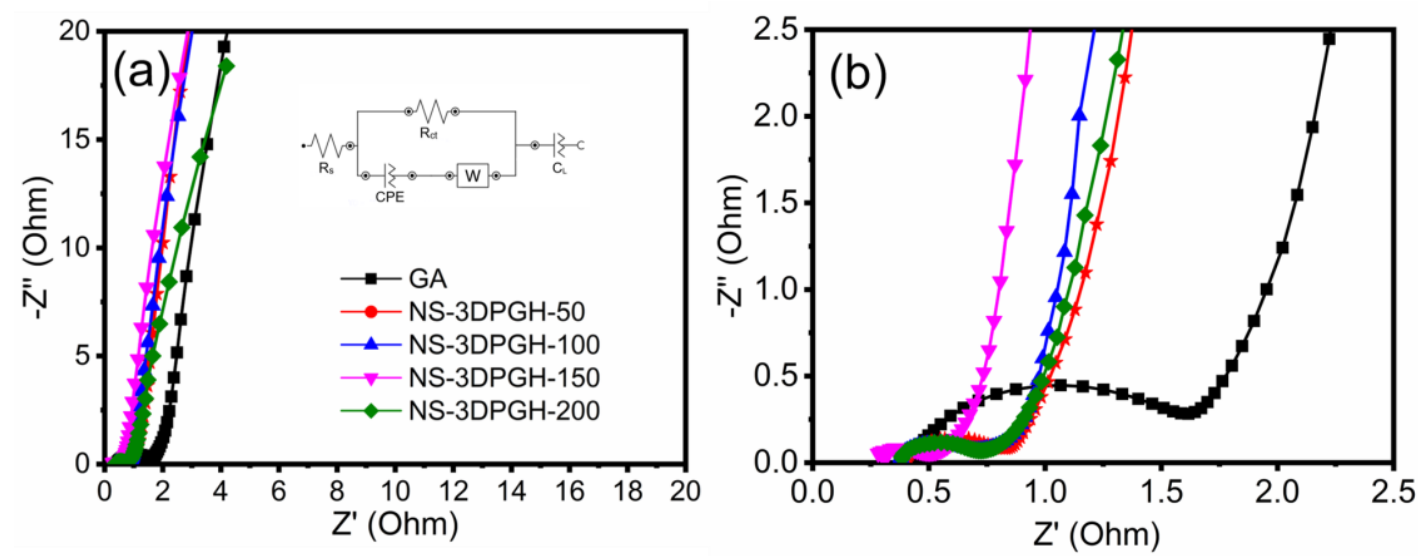

Figure 7. (a) Nyquist diagrams of undoped GA and the NS-3DPGHs electrodes. The equivalent circuit for fitting the resulting Nyquist diagrams are displayed in the illustration. (b) Enlarged Nyquist diagrams of the high-frequency region.

To get an advanced investigation of the electrochemical performance of NS-3DPGHs, EIS measurements of all samples were performed. Figure 7 displays the Nyquist plots during all NS-3DPGHs and GA. The nearly theoretical capacitive performance is represented in the low-frequency section which has a steep linear curve. The equivalent circuit is displayed in the illustration of Figure $7 \mathrm{a}$, where $R_{S}$ is the equivalent series resistant, which represents the impedance associated with the purely Ohmic contribution of electrodes and electrolyte in the high-frequency region [37]. For GA, NS-3DPGH-50, NS-3DPGH-100, NS-3DPGH-150 and NS-3DPGH-200, the $R_{S}$ values are $0.43,0.41,0.36,0.22$ and $0.37 \Omega$, respectively. The NS-3DPGH-150 has the smallest $R_{s}$, suggesting the lowest internal resistance. Fitting 
curve is displayed in the Figure S3. The semi-circle in the middle high-frequency section is a measure of the charge transfer resistant $\left(R_{c t}\right)$ associated with Faradaic (pseudocapacitive) reactions. In Figure $7 \mathrm{~b}$, the $R_{c t}$ values of GA and NS-3DPGHs are $1.35,0.50,0.49,0.40$ and $0.48 \Omega$, respectively. Compared with GA electrode, the lower $R_{s}$ and $R_{c t}$ of NS-3DPGHs electrode are attributed to augment the intense polarization of the carbon-based surface after nitrogen and sulfur doping [38, 39]. The results of EIS further show that NS-3DPGH-150, with the lowest $R_{c t}$ of $0.40 \Omega$, has the best electrochemical performance.
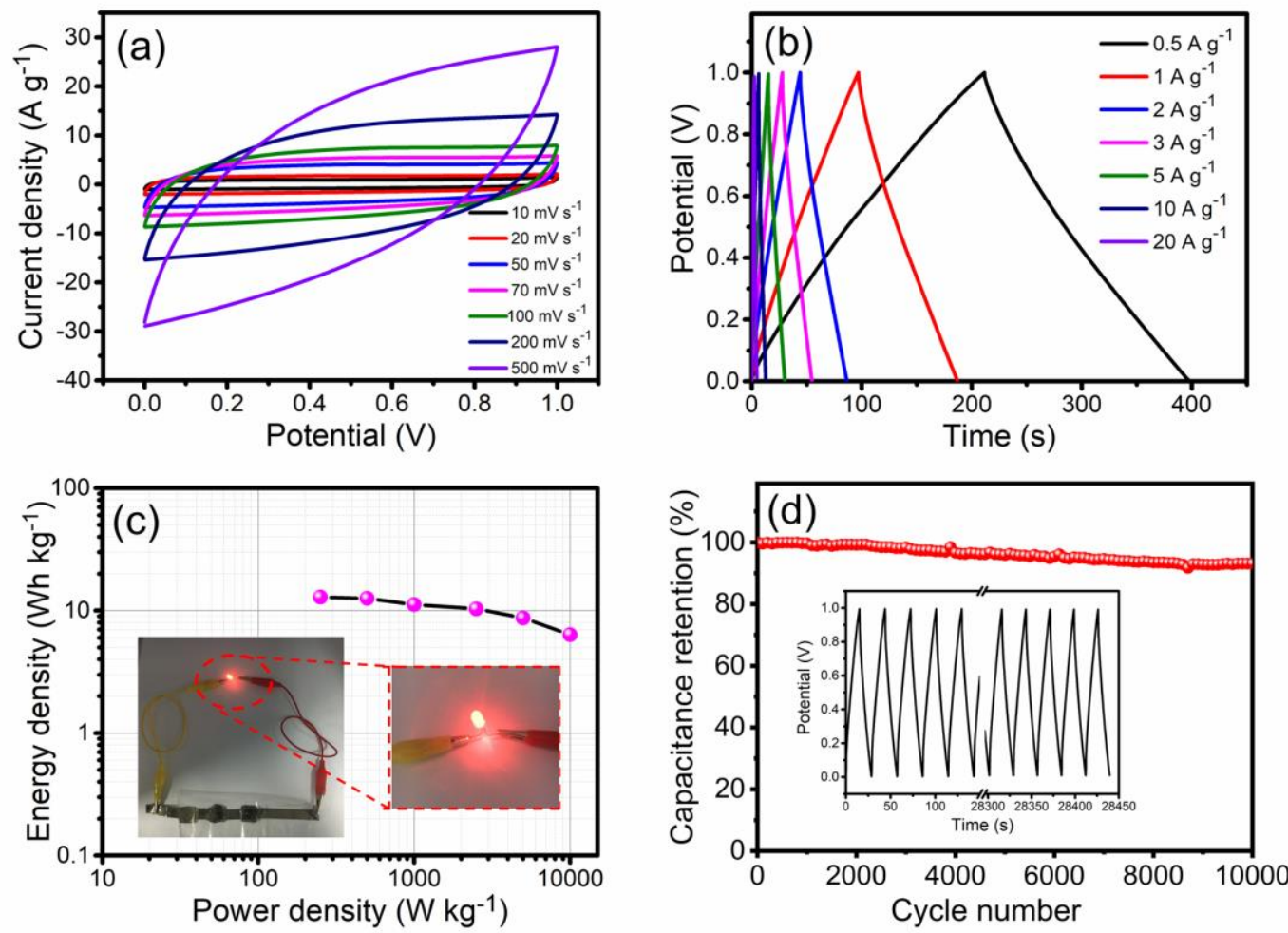

Figure 8. Electrochemical performance of the NS-3DPGH-150 symmetric SCs tested in a two-electrode system in $6 \mathrm{M} \mathrm{KOH}$. (a) The $\mathrm{CV}$ curves at different scan rates from $10 \mathrm{mV} \mathrm{s}^{-1}$ to $500 \mathrm{mV} \mathrm{s}^{-1}$. (b) The GCD curves from $0.5 \mathrm{~A} \mathrm{~g}^{-1}$ to $20 \mathrm{~A} \mathrm{~g}^{-1}$. (c) The Ragone plot of the symmetric SCs. Inset: optical images showing the illumination of an LED when three cells are connected in electrical series. (d) Cycling durability test measured for 10000 cycles at $5 \mathrm{Ag} \mathrm{g}^{-1}$. 
To better survey the practical performance of the NS-3DPGH-150 electrode, a SC was assembled via employing two NS-3DPGH-150 electrodes. The scan rates, ranging from 10 to $500 \mathrm{mV} \mathrm{s}^{-1}$, are illustrated through the $\mathrm{CV}$ curves of the assembled symmetric supercapacitor in Figure 8a. All curves present relatively rectangular shapes, which even scan rate is up to $500 \mathrm{mV} \mathrm{s}^{-1}$, indicating that the electrode has extraordinary rate performance. The device GCD curves assembled using NS-3DPGH-150 is displayed in Figure 8b. The highly symmetric and linear curves are indicative of effective electrolyte ion transport throughout the electrode and ideal electrical DL capacitive behavior. Calculated specific capacitance of the assembled device is $92.9 \mathrm{~F} \mathrm{~g}^{-1}$ at $0.5 \mathrm{~A} \mathrm{~g}^{-1}$ and remains at $45.8 \mathrm{~F} \mathrm{~g}^{-1}$ even at a high current density of $20 \mathrm{~A} \mathrm{~g}^{-1}$. According to the Ragone plot in Figure 8c, with the $249 \mathrm{~W} \mathrm{~kg}^{-1}$ power density, the maximal energy density achieved is $12.9 \mathrm{Wh} \mathrm{kg}^{-1}$; it is higher than the majority of previously reported symmetrical supercapacitor in the similar electrode materials case (Table S3).

To demonstrate practical relevance, three supercapacitor units were connected in the identical series which make it convenient to activate a red LED after being charged (inserts of Figure 8c), which implies that the fabricated supercapacitor facility, with practical function, it can be applied to the real factory or similar place. Moreover, under the condition of the long-term cycling stability of the device in which the current density can be measured as $5 \mathrm{~A} \mathrm{~g}^{-1}$, with the interminable cycles finishing, 93.1\% of initial capacitance can still retain when the amount of the cycles is up to ten thousand (Figure 8d). These results confirm that unique NS-3DPGH material has a potential practical application.

\section{Conclusion}

The $\mathrm{N}$ and $\mathrm{S}$ co-doped graphene aerogel has been fabricated through a 
hydrothermal method through chemical reduction of the GO with thiourea, for application in supercapacitor electrodes. The obtained NS-3DPGH materials exhibit a three-dimensional porous structure, the formed mesopores abridge ion diffusion path and promote the entry of electrolyte ion to get excellent electrochemical performance. NS-3DPGH-150 shows superior electrochemical performance, including a senior specific capacitance of $412.9 \mathrm{~F} \mathrm{~g}^{-1}$ at $0.5 \mathrm{~A} \mathrm{~g}^{-1}$ and $96.4 \%$ capacitance retention after 10000 cycles. A symmetric supercapacitor based on NS-3DPGH-150 was found to deliver an enormous energy density of $12.9 \mathrm{Wh} \mathrm{kg}^{-1}$, in which power density is as much as $249 \mathrm{~W} \mathrm{~kg}^{-1}$. The as-prepared materials have been proven to be prospect for supercapacitor applications. However, the merits of this class of material point to beneficial utilization in other energy conversion and storage systems.

\section{Acknowledgments}

This work was supported by the NSFC (Nos. 11504229, 51602193), Shanghai "Chen Guang" project (16CG63). Open project of Key Laboratory of Artificial Structures and Quantum Control (Ministry of Education), Shanghai Jiao Tong University. Shanghai University of Engineering Science Innovation Fund for Graduate Students (17KY0512). The Talent Program of Shanghai University of Engineering Science, and the ESI Program of Shanghai University of Engineering Science (ESI201809, ESI201802). GA, PRS and DJLB acknowledge funding from the EPSRC (EP/R023581/1, EP/P009050/1).

\section{References:}

[1] Q. X. Zhou, W. W. Ju, Y. L. Yong, Q. Zhang, Y. L. Liu, J. L. Li, Effect of the $\mathrm{N} / \mathrm{P} / \mathrm{S}$ and transition-metal co-doping on the quantum capacitance of supercapacitor electrodes based on mono- and multilayer graphene, Carbon, 170 (2020) 368-379.

[2] W. Y. Li, B. J. Zhang, R. J. Lin, S. M. Ho-Kimura, G. J. He, X. Y. Zhou, J. Q. Hu, 
I. P. Parkin, A dendritic nickel cobalt sulfide nanostructure for alkaline battery electrodes, Adv. Funct. Mater., 28 (2018) 1705937.

[3] H. Q. Gao, D. Zhang, H. T. Zhou, J. C. Wu, G. J. Xu, Z. L. Huang, M. H. Liu, J. H. Yang, D. Chen, Boosting gravimetric and volumetric energy density of supercapacitors by 3D pomegranate-like porous carbon structure design, Appl. Surf. Sci., 534 (2020) 147613.

[4] W. T. Wang, L. S. Lu, L. S. Lu, Y. X. Xie, X. K. Mei, Y. Tang, W. B. Wu, R. X. Liang, Tailoring the surface morphology and nanoparticle distribution of laser-induced graphene/ $\mathrm{Co}_{3} \mathrm{O}_{4}$ for high-performance flexible microsupercapacitors, Appl. Surf. Sci., 504 (2020) 144487.

[5] S. Korkmaz, I. A. Kariper, Graphene and graphene oxide based aerogels: Synthesis, characteristics and supercapacitor applications, J. Energy Storage, 27 (2020) 101038.

[6] L. Miao, H. Duan, Z. W. Wang, Y. K. Lv, W. Xiong, D. Z. Zhu, L. H. Gan, L. C. Li, M. X. Liu, Improving the pore-ion size compatibility between poly (ionic liquid)-derived carbons and high-voltage electrolytes for high energy-power supercapacitors, Chem. Eng. J., 382 (2019) 122945.

[7] C. Y. Xiong, B.B. Li, X. Lin, H. G. Liu, Y. J. Xu, J. J. Mao, C. Duan, T. H. Li, Y. H. $\mathrm{Ni}$, The recent progress on three-dimensional porous graphene-based hybrid structure for supercapacitor, Compos. B. Eng., 165 (2019) 10-46.

[8] D. L. Wu, T. Wang, L. X. Wang, D. Z. Jia, Hydrothermal synthesis of nitrogen, sulfur co-doped graphene and its high performance in supercapacitor and oxygen reduction reaction, Microporous Mesoporous Mater., 290 (2019) 109556.

[9] A. Ramadan, M. Anas, S. Ebrahim, M. Soliman, A. A. Aly, Effect of Co-doped graphene quantum dots to polyaniline ratio on performance of supercapacitor. J. Mater. 
Sci.-Mater. Electron., 12 (2020) 7247-7259.

[10] L. J. Zhang, S. W. Or, Self-assembled three-dimensional macroscopic graphene/MXene-based hydrogel as electrode for supercapacitor, Appl. Mater., 8 (2020) 091101.

[11] X. Zhang, Q. Y. Fan, H. Yang, A. M. Liu, Graphene oxide template-directed synthesis of porous carbon nanosheets from expired wheat flour for high-performance supercapacitors, New J. Chem., 42 (2018) 11689.

[12] S. Jia, J. Wei, X. T. Meng, Z. Q. Shao, Facile and friendly preparation of N/S Co-doped graphene-like carbon nanosheets with hierarchical pore by molten salt for all-solid-state supercapacitor, Electrochimi. Acta, 331 (2020) 135338.

[13] J. L. Liu, Y. R. Zhu, X. H, Chen, W. J. Yi, Nitrogen, sulfur and phosphorus tri-doped holey graphene oxide as a novel electrode material for application in supercapacitor, J. Alloys Compd., 815 (2020) 152328.

[14] G. Arthi, D. R. Tejas, B. Sushmee, Green synthesis of nitrogen, sulfur-co-doped worm-like hierarchical porous carbon derived from ginger for outstanding supercapacitor performance, Carbon, 168 (2020) 209-219.

[15] R. Nankya, D. O. Opar, M. J. Kim, S. M. Paek, H. Jung, Synergetic effect of nitrogen and sulfur co-doping in mesoporous graphene for enhanced energy storage properties in supercapacitors and lithium-ion batteries, J. Solid Stata Chem., 289 (2020) 121451.

[16] A. Ghazitabar, M. Naderi, D. F. Haghshenas, M. Rezaei, Synthesis of N-Doped Graphene Aerogel/ $\mathrm{Co}_{3} \mathrm{O}_{4} / \mathrm{ZnO}$ Ternary Nanocomposite via Mild Reduction Method with an Emphasis on Its Electrochemical Characteristics. J. Alloys Compd., 794 (2019) $625-633$

[17] W. J. Zhang, Z. T. Chen, X. L. Guo, K. Jin, Y. X. Wang, L. Li, Y. Zhang, Z. M. 
Wang, L. T. Sun, T. Zhang, N/S co-doped three-dimensional graphene hydrogel for high performance supercapacitor, Electrochimi. Acta, 278 (2018) 51-60.

[18] L. Chen, Z. Y. Wen, L. N. Chen, W. P. Wang, Q. Ai, G. M. Hou, Y. H. Li, J. Lou, L. J. Ci, Nitrogen and sulfur co-doped porous carbon fibers film for flexible symmetric all-solid-state supercapacitors, Carbon, 158 (2020) 456-464.

[19] G. Arthi, B. Sushmee, Sulfonated porous carbon nanosheets derived from oak nutshell based high-performance supercapacitor for powering electronic devices, Renew. Energ., 161 (2020) 173-183.

[20] Z. Q. Li, W. X. He, X. X. Wang, X. L. Wang, M. Song, J. L. Zhao, N/S dual-doped graphene with high defect density for enhanced supercapacitor properties, Int. J. Hydrog. Energy, 45 (2020) 112-122.

[21] X. Zhang, Q. Y. Fan, N. Qu, H. Yang, M. Wang, A. M. Liu, J. Yang, Ultrathin 2D nitrogen-doped carbon nanosheets for high performance supercapacitors: insight into the effects of graphene oxides, Nanoscale, 11 (2019) 8588.

[22] L. L. Cheng, Y. Y. Hu, D. D. Qiao, Y. Zhu, H. Wang, Z. Jiao, One-step radiolytic synthesis of heteroatom ( $\mathrm{N}$ and $\mathrm{S}$ ) co-doped graphene for supercapacitors, Electrochimi. Acta, 259 (2018) 587-597.

[23] J. H. Li, G. P. Zhang, C. P. Fu, L. B. Deng, R. Sun, C. P. Wong, Facile preparation of nitrogen/sulfur co-doped and hierarchical porous graphene hydrogel for high-performance electrochemical capacitor, J. Power Sources, 345 (2017) 146-155.

[24] A. Ghazitabar, M. Naderi, D. Fatmehsari Haghshenas, A facile chemical route for synthesis of nitrogen-doped graphene aerogel decorated by $\mathrm{Co}_{3} \mathrm{O}_{4}$ nanoparticles, Ceram. Int., 44 (2018) 23162-23171.

[25] M. Q. Liu, S. L. Huo, M. Xu, L. L. Wu, M. L. Liu, Y. F. Xue, Y. M. Yan, Structural engineering of N/S co-doped carbon material as high-performance 
electrode for supercapacitors, Electrochimi. Acta, 274 (2018) 389-399.

[26] Z. Q. Li, W. X. He, X. X. Wang, X. L. Wang, M. Song, J. L. Zhao, N/S dual-doped graphene with high defect density for enhanced supercapacitor properties, Int. J. Hydrog. Energy, 45 (2019) 112-122.

[27] H. G. Wei, H. Wang, A. Li, H. Q. Li, D. P. Cui, M. Y. Dong, J. Lin, J. C. Fan, J. X. Zhang, H. Hou, Advanced porous hierarchical activated carbon derived from agricultural wastes toward high performance supercapacitors, J. Alloys Compd., 820 (2019) 15311.

[28] M. Wang, J. Yang, S. Y. Liu, M. Z. Li, C. Hu, J. S. Qiu, Nitrogen-doped hierarchically porous carbon nanosheets derived from polymerigraphene oxide hydrogels for high-performance supercapacitors, J. Colloid Interface Sci., 560 (2019) 69-76.

[29] R. Shadkam, M. Naderi, A. Ghazitabar, A. Asghari-Alamadri, S. Shateri, Enhanced electrochemical performance of graphene aerogels by using combined reducing agents based on mild chemical reduction method. Ceram. Int., 46 (2020) 22197-22207.

[30] B. B. Guo, R. G. Ma, Z. C. Li, S. K. Guo, J. Luo, M. H. Yang, Q. Liu, T. Thomas, J. C. Wang, Hierarchical N-Doped Porous Carbons for Zn-Air Batteries and Supercapacitors, Nanomicro Lett., 12 (2020) 20.

[31] Q. Lv, H. L. Hao, M. M. Ge, W. Y. Li, S-doped graphene/mixed-valent manganese oxides composite electrode with superior performance for supercapacitors, J. Alloys Compd., 819 (2019) 132970.

[32] C. H. Ruan, Y. B. Xie, Electrochemical performance of activated carbon fiber with hydrogen bond-induced high sulfur/nitrogen doping, RSC Advances, 10 (2020) 37631-37643. 
[33] S. G. Dai, Z. Liu, B. T. Zhao, J. H. Zeng, H. Hu, Q. B. Zhang, D. C. Chen, C. Qu, D. Dang, M. L. Liu, A high-performance supercapacitor electrode based on N-doped porous graphene, J. Power Sources, 387 (2018) 43-48.

[34] W. L. Zhang, C. Xu, C. Q. Ma, G. X. Li, Y. Z. Wang, K. Y. Zhang, F. Li, C. Liu, H. M. Cheng, Y. W. Du, N. J. Tang, W. C. Ren, Nitrogen-Superdoped 3D Graphene Networks for High-Performance Supercapacitors, Adv. Mater., 29 (2017) 26.

[35] M. Y. Emran, M. A. Shenashen, A. A. Abdelwabab, M. Abdelmottaleb, S. A. E. Safty, Facile synthesis of microporous sulfur-doped carbon spheres as electrodes for ultrasensitive detection of ascorbic acid in food and pharmaceutical products, New J. Chem., 7 (2018) 5037-5044.

[36] A. Gopalakrishnan, S. Badhulika, Ultrathin graphene-like 2D porous carbon nanosheets and its excellent capacitance retention for supercapacitor, J. Ind. Eng. Chem., 6 (2018) 257-266.

[37] X. Zhao, Ming. Li, H. W. Dong, Y. L. Liu, H. Hu, Y. J. Cai, Y. Liang, Y. Xiao, M. T. Zheng, Interconnected 3D Network of Carbon Dots decorated Reduced Graphene Oxide Nanosheets for High-Performance Supercapacitor, ChemSusChem, 10 (2017) 2626-2634.

[38] L. L. Zhang, H. X. Chen, X. Y. Lu, Y. Wang, L. L. Tan, D. P. Sui, W. Qi, Fabrication of N, S co-doped graphene aerogel for high-performance supercapacitors: $\pi$-conjugated planar molecules as efficient dopants and pillared agents, Appl. Surf. Sci., 529 (2020) 147022.

[39] B. T. Zhao, D. C. Chen, X. H. Xiong, B. Song, R. Z. Hu, Q. B. Zhang, B. H. Rainwater, G. H. Waller, D. X. Zhen, Y. Ding, A high-energy, long cycle-life hybrid supercapacitor based on graphene composite electrodes, Energy Storage Mater., 7 (2017) 32-39. 